\title{
Interacting with radio telescopes in real-time during VLBI sessions using e-control
}

\author{
A. Neidhardt;, M. Ettl \\ Forschungseinrichtung Satellitengeodäsie, Technische Universität München, \\ Geodätisches Observatorium Wettzell \\ Sackenrieder Str. 25, D-93444 Bad Kötzting, Germany \\ C. Plötz, M. Mühlbauer, H. Hase \\ Bundesamt für Kartographie und Geodäsie, Geodätisches Observatorium Wettzell \\ Sackenrieder Str. 25, D-93444 Bad Kötzting, Germany
}

\author{
S. Sobarzo, C. Herrera \\ Universidad de Concepción \\ Camino Einstein Km 2,5., Casilla 4036, Correo 3, Concepción, Chile
}

\section{W. Alef, H. Rottmann}

Max-Planck-Institut für Radioastromonie

Auf dem Hügel 69, 53121 Bonn, Germany

\section{E. Himwich}

National Aeronautics and Space Administration/Goddard Space Flight Center

Greenbelt, MD 20771, USA

In times of e-VLBI, a live monitoring of system states or system health and a dedicated control access by session schedulers can increase the efficiency of observations. At the Geodetic Observatory Wettzell a software extension to the existing NASA Field System has been developed for remote control of telescope observations in cooperation with the Max-Planck-Institute for Radio Astronomy in Bonn. It uses the principle of a remotely accessible, autonomous process cell as server extension to the Field System. The communication is realized also for low transfer rates on the basis of Remote Procedure Calls (RPC) and uses generative programming with the interface software generator idl2rpc.pl developed at Wettzell. The user interacts with this system over a modern graphical user interface realized with wxWidgets. For security reasons the communication is automatically tunneled through a Secure Shell (SSH) session to the telescope. After successful test observations with the telescopes at O'Higgins, Concepción, and Wettzell and while it is already regularly used at Wettzell, future developments for safe and secure authentication and user role management procedures will be realized within the upcoming NEXPRES project.

The 10th European VLBI Network Symposium and EVN Users Meeting: VLBI and the new generation of radio arrays

September 20th - 24th, 2010

Manchester, $U K$

\footnotetext{
*Speaker.
} 


\section{Introduction}

Personnel from the Geodetic Observatory Wettzell operate not only the 20 meter radio telescope at Wettzell. In cooperation with other institutes they also run the 9 meter radio telescope at the German Antarctic Receiving Station (GARS) O'Higgins, Antarctica and the 6 meter radio telescope of the Transportable Integrated Geodetic Observatory (TIGO) Concepción, Chile for geodetic VLBI experiments. In near future also the new build TWIN radio telescopes at Wettzell must be operated in parallel with the same staff. Therefore the team at the Geodetic Observatory investigates different observation control strategies to operate this world-wide network of telescopes. Remote access or unattended modes are tested and are already in partial used. They allow different control centers and responsible operators to share the control of the telescopes.

\section{New operation control strategies}

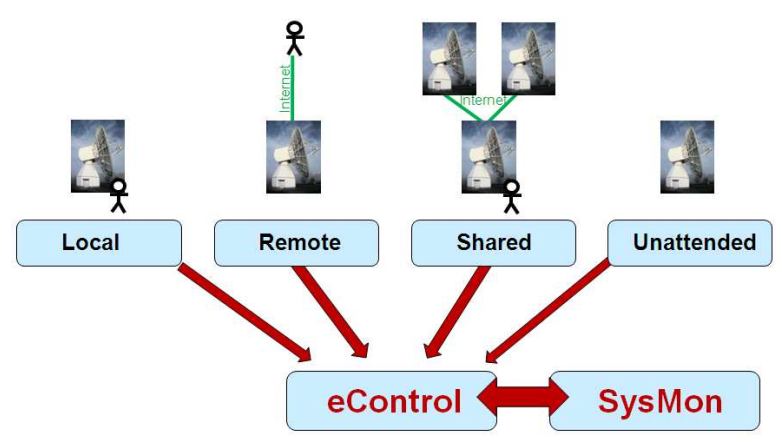

Figure 1: The new operation control strategies.

The standard case to control operations is that an observer controls a VLBI session locally on site at the telescope (local observation). He has direct access to the control system, the hardware and the telescope itself. In case of an error the operator can directly interact and stop the system manually. It is important that well educated personnel can detect problems immediately by using all human senses. But this operation mode is also the most time consuming one, because 3 shifts in 24 hours seven days a week must be planned.

With new remote control technologies it is no longer necessary for the operator to be on location (see fig. 1). The operator can control the system from remote (remote observation). On stable internet connections and with intelligent and self-controlling mechanisms on location of the radio telescopes critical situations can automatically be detected. Autonomous, redundant systems can check system states and stop the operation in case of an error. The important system information can be transfered to responsible operators over the internet to each place all over the world. As the operator just gets the information which is offered by the system, an additional monitoring on location of the telescope must replace the senses of a local operator. These monitoring data allow the automatic detection of critical states and situations, either automatically taking action or informing the remote operator.

This technology can also be used to control more than one telescope by one operator. The control can be shared between different operators on different sites (shared observation). Hierar- 
chically arranged control structures are needed to give one operator the possibility to attend and control several parallel observations on different sites by remote. A sophisticated graphical user interaction is as important as reliable communication systems. Communication losses must be detected, reestablished and proofed. During blackouts the systems must be able to run autonomously.

At Wettzell also completely unattended observations have been done especially for the weekend sessions for over 2 years now. For these the antenna runs completely autonomous and automatic without an operator (unattended observation). Well maintained and stable telescopes are needed. It is also helpful to have an on-call service, where especially trained staff can be activated on time to react on critcial, automatically not solvable situations. The most important implementationof the system is to avoid such situations is a functional and detailed system monitoring.

These remote and shared observations offer many possibilities: A passive data access can be granted for live monitoring. There are possibilities for tele-working with full control access or specialists can assist local operators by remote. Very remote telescopes such as those in Antarctica can be controlled from large distances. Further, shared observations can reduce the manpower for shifts or help react to current research needs.

\section{A software dealing with the needs: e-control}

Because of the remote locations especially in case of the telescope in the Antarctica a first concept was developed, to control sites remotely on the basis of the current equipment, which is controlled by the NASA Field System (FS) software package. Therefore Wettzell is developing a software extension for remote control to the existing NASA Field System (FS) in cooperation with the Max-Planck-Institute for Radio Astronomy (Bonn). The software uses remotely accessible, autonomous control cells as server extension[3] to the FS on the basis of Remote Procedure Calls (RPC) (see [1]).

This is a very stable, well known and well supported communication package. As already existing tools to forward mouse, keyboard and video signals are suboptimal, because they don't allow monitoring of the internet connection itself to facilitate safety actions the new software offers an Ethernet based, safe and stable remote communication. Since most of the new devices controlled by the field system are also connected via ethernet mechanisms the new concept also includes ideas to standardize such individual communication needs. Together it offers the appropriate elements for remote control as a new VLBI observation mode, possibly named "e-control".

\section{The layers of e-control as extension of the NASA field System}

The given remote control design realises a classic client-server-model. In the case of the FS an additional, automatically created communication is defined. The client-server-based complete stack for e-control from user interaction via remote procedure call communication to the FS interaction is shown in fig. 2. One of the main drivers in the given design is a strict separation of control, communication and presentation logic. The complete arithmetic and workflow control logic reside in the server, defined as device control code. It is an autonomous working process which interacts with the remote controlled device (here at this level, the FS). The communication code indepen- 


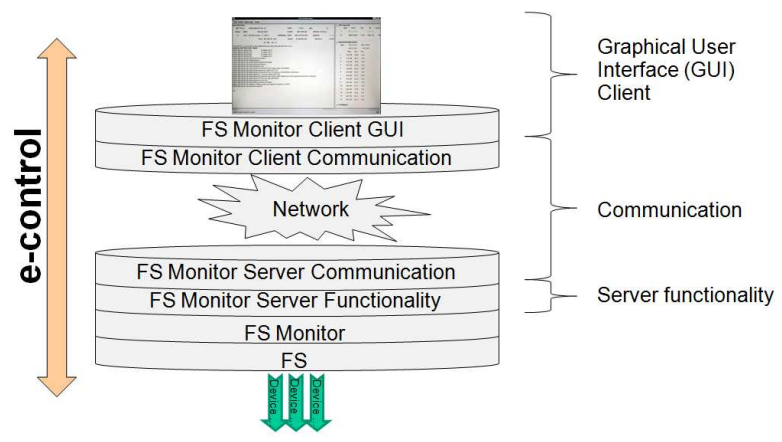

Figure 2: The complete e-control stack.

dently connects the server to the outer world for requesting clients. The clients are only used to realize an user interface with a presentation of the server processed elements.

In the first implementation the communication consists only of a few methods returning the local information output as string arrays to the remote requesting client. Therefore an additional $\mathrm{C}$-written adapter (FS monitor) allows the connection to the FS via shared memory access. The client can also send a string command to the server. The commands are injected into FS using the supported injection methods. To smooth the communication behaviour the server uses threads to separate between the asynchronous remote procedure calls and the contact with the FS. Semaphore protected variables allow the handling of critical sections when both tasks work in parallel with the same variable values.

Overall the created server acts completely autonomously. It can be used to check system status information independently and can decide what to do to keep stable and safe states. Such controlling utilities can be defined as autonomous process cells. The generated watchdog process keeps it alive and an automatic safety device allows to register if a responsible client is connected. After a breakdown of the communication to the client the server can operate completely autonomously until a critical situation (e.g. increase of wind speed to a level which is critical for the telescope) forces it to run into a safe state. In combination with additional monitoring information around the site (meteorology, power supply, air conditioning status, etc.) that compact server extends the FS for a reliable remote control.

\section{The middleware generator "idl2rpc.pl"}

To realize the communication a new middleware generator "idl2rpc.pl" was designed. This generator itself is just a single Perl script which uses only the Open Network Computing Remote Procedure Call (ONC RPC) realisations of standard Linux distributions. It extends "rpcgen". Several $\mathrm{C}++$ adaptor classes for the $\mathrm{C}$ written RPC communication are created. They can directly be used in the application code. Dedicated modules offer threads to use in parallel tasks with semaphores to protect critical sections. A sophisticated communication control mechanisms, such as a watchdog process, which e.g. always restarts the server after an unexpected crash, extends the stability. An Automatic Safety Device (ASD) controls the existence of a responsible client and forces safety actions when the connection to the client breaks down[3]. 


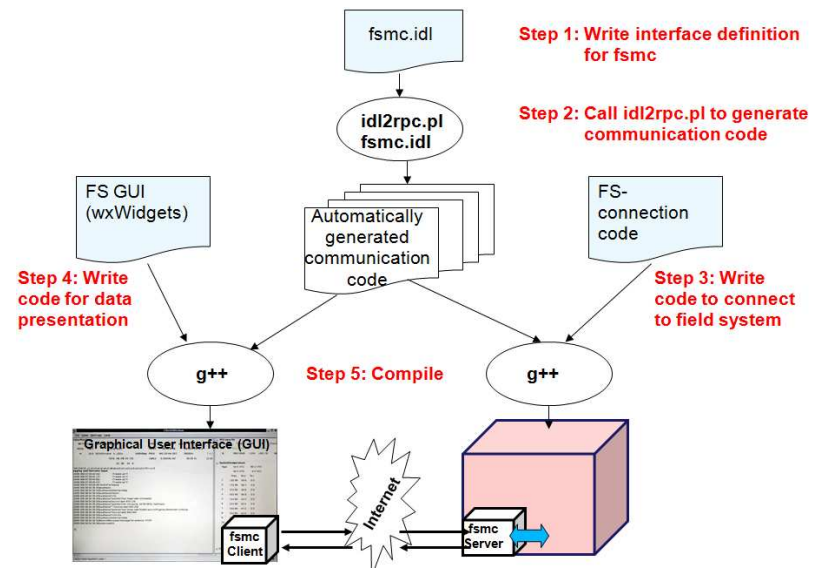

Figure 3: The generation process using the code generator "idl2rpc.pl".

"idl2rpc.pl" reads an interface definition file and produces all of the necessary modules. In only a few steps the complete communication is generated with this light generator (see fig. 3). It is open source and no additional, external packages are needed, for example after an upgrade of the operating system. Because of the usage of the Transmission Control Protocol over Internet Protocol (TCP/IP) or the User Datagram Protocol over Internet Protocol (UDP/IP) without additional, proprietary layers the generated communication can easily tunnel firewall barriers.

This helps to develope distributed systems, consisting of several independent computers (processors), which are connected together to solve a collective task in a cooperative way. During the processing time they don't share memory, clocks or other hardware and just communicate information while transferring messages via a computer network ([5] and [6]). Each hardware device can be represented as server which is accessible via a generated communication. Other servers can contain several clients to access and autonomously control different, subordinated devices. These combination servers themselves again offer services on a generated communication. In summary a hierarchical architecture is realized which establishes hierarchical and autonomous control zones. On the upper end of this hierarchy an user interface allows human interaction.

All of the servers on one platform can be found via registrations in a "portmapper", where all services and their ports are registered. It is also possible to directly contact the servers on the dedicated ports. But the servers can also be distributed on several platforms. The generation method therefor offers the basic and very flexible skeleton to program the different elements of this distributed system, where clients and servers can be individually defined.

To bring in a level of security the Secure Shell (SSH) tunneling methods with its several authentication possibilities can be used to build up an access protection with automatic connection control. This "sshbroker" (re-)establishes broken SSH tunnel without user interaction.

\section{The new graphical user interface for remote control of the NASA field System}

On the other end of the remote control the operator interacts with the system. It is possible to implement command line clients as well as highly sophisticated web applications or graphical user interfaces. This permits management of devices remotely via browser, command line and/or graph- 
ical user interface (GUI). For the described FS extension wxWidgets is the prefered way to offer a GUI. wxWidgets is a $\mathrm{C}++$ based open source framework for platform independent developments of graphical user interfaces [7]. Although the current RPC generator only supports Linux systems (32 and $64 \mathrm{Bit}$ ), the graphical user interface is modular enough to support different platforms like Windows, Linux, OSX and others. In terms of the proposed FS extension a new graphical user interface was created using the wxWidgets framework for its realisation. To keep usage similar, the display elements are organized to be like those of the current local interface to the FS.

\section{Remote control tests}

Based on this technology, several remote control and attendance tests were successfully shown with telescopes in Germany, Chile and Antarctica. The latest test now demonstrated the idea of shared observations. Both telescopes at Wettzell, in Germany and Concepción, Chile were operated by one operator at Wettzell for a whole 24 hour IVS-session (R4438), where both telescopes participated (see [2]). For security reasons, the whole communication was tunneled using SSH. During the session, local operators were available on both sides in case of malfunctions or for assistance. The integrated chat functionality and a webcam live view gave additional feedback.

\section{Summary and outlook}

Overall the described method allows the development of distributed systems consisting of several independent servers which act completely autonomously. It extends given structures to have a remote control possibility and splits complex systems up into several manageable units interacting together with a general, standardized but also flexible communication method.

The resulting software is an option for upcoming Fundamental Stations with several different colocated measuring systems like radio telescopes and laser ranging systems to realize remotely controllable, autonomous subsystems especially along the goals of the Global Geodetic Observing System (GGOS). The method could also be interesting as pathfinder technology for upcoming Square Kilimeter Arrays (SKA).

The new observing strategies for VLBI2010 on the basis of remote and shared observations can also be realised with very remote stations. But nevertheless there are always some situations which cannot be controlled and handled by such an automated system (as access locks after power failures), so that responsible, well educated engineers at the sites should always be the final instance of automation.

Future developments for safe and secure authentication and user role management procedures will be realized within the upcoming Novel EXplorations Pushing Robust e-VLBI Services (NEXPRES) project during the following years (see [4]). According to this a new standardized system monitoring will be designed and realized within the same project. It is a part of the work package 5 in the EU financed project as developement of the Forschungseinrichtung Satellitengeodäsie, Technische Universität München together with Max-Planck-Institute for Radioastronomy (MPIfR) Bonn. 


\section{References}

[1] J. Bloomer, Power programming with RPC, O'Reilly and Associates, Inc., ISBN: 0-937175-77-3 (1992).

[2] M. Ettl, A. Neidhardt, M. Mühlbauer, C. Plötz, H. Hase, S. Sobarzo, C. Herrera, E. Oñate, P. Zaror, F. Pedreros, O. Zapato, First proof of concept of remote attendance for future observation strategies between Wettzell (Germany) and Concepcion (Chile), 10th European VLBI Network Symposium and EVN Users Meeting: VLBI and the new generation of radio arrays, POS (10th EVN Symposium) 075 (2010).

[3] A. Neidhardt, M. Ettl, C. Plötz, M. Mühlbauer, R. Dassing, H. Hase, S. Sobarzo, C. Herrera, W. Alef, $\mathrm{H}$. Rottmann, e-control: new concepts for remote control of VLBI-telescopes and first experiences at Wettzell, The 8th International e-VLBI Workshop, EXPReS09, PoS (EXPReS 09 ) 038 (2009).

[4] NEXPReS, Novel EXplorations Pushing Robust e-VLBI Services (NEXPRES), http: //www. nexpres.eu/ (2010).

[5] M. Singhal, N. G. Shivaratri, Advanced Concepts in Operating Systems, McGraw-Hill, Inc. (1994).

[6] A. Puder, K. Römer, Middleware für verteilte Systeme, dpunkt-Verlag GmbH (2001).

[7] J. Smart, K. Hock, S. Csomor, Cross-Platform GUI Programming with wxWidgets, Prentice Hall International (2005). 